\title{
Uterine Sub Mucosal Leiomyoma (Fibroid) - A Case Report
}

\author{
Sunita Nayak ${ }^{1}$, Suren Prasad Dash ${ }^{2}$, Muktikanta Khatua ${ }^{3}$ \\ ${ }^{I}$ Tutor, Department of Anatomy, SCB Medical College, Cuttack, Odisha, India \\ ${ }^{2}$ Consultant Radiologist, Sahara Diagnostics, Courtpeta Square, Berhampur, Odisha, India \\ ${ }^{3}$ Assistant Surgeon, District Head Quarter Hospital, Koraput, Odisha, India
}

\begin{abstract}
Submucosal fibroids are the least common of the various types of fibroid tumors. These fibroids develop just under the lining of the uterine cavity. Large submucosal fibroid tumors may increase the size of the uterine cavity and can block the fallopian tubes. This can cause complications if one is trying to get pregnant. Some fibroids do not produce any symptoms at all while others can be severely symptomatic. Associated symptoms with submucosal fibroids include very heavy menstrual bleeding or excessive and prolonged menstruation. The passage of clots and frequent soiling can take its toll on one's everyday lifestyle. Untreated, prolonged or excessive bleeding can cause more complicated problems such as fatigue or anemia which could potentially lead to a need for blood transfusions. This case report is about a 44 years old lady having two kids, complaining of very heavy menstrual bleeding, pelvic pain and an enlarged abdomen.
\end{abstract}

Keywords: Fibroid, Fibroleiomyoma, Fibromyoma, Myoma, Submucosal, Uterine leiomyoma

\section{Introduction}

Uterine fibroids, also known as leiomyomata or myomas, are benign (noncancerous) tumors of the muscular wall of the uterus. They are the most common tumor of the female genital tract and affect 1 in 3 women older than 35 years of age. Fibroids are classified according to their location within the uterine wall (Fig. 1). Submucous or submucosal fibroids occur just under the endometrial lining of the uterine cavity. Intramural fibroids occur in the muscular wall. Subserosal fibroids occur under the outside covering of the uterus. As fibroids enlarge, the distinction between the types becomes blurred. Pedunculated fibroids occur on a stalk, project from the surface of the uterus and can be confused with ovarian masses. They can project from the inner lining of the uterus and even extend through the cervix.



Fig. 1: Schematic drawing of various types of uterine fibroids; $a=$ subserosal fibroids, $b=$ intramural fibroids, $\mathrm{c}=$ submucosal fibroid, $\mathrm{d}=$ pedunculated submucosal fibroid, $\mathrm{e}=$ fibroid in statu nascendi, $\mathrm{f}=$ fibroid of the broad ligament

\section{Case Presentation}

A 44 years old lady having two kids came with the chief complaint of excessive menstrual bleeding with clots, pelvic pain, incontinence and prolonged monthly periods. She had an enlarged abdomen which can be mistaken for bloating, weight gain or pregnancy. She also complained of fatigue. In spite of repeated treatment with hormone therapy, there was no response. Investigations report include: Hemogram $=9.8 \mathrm{gm} \%$, Total $\mathrm{RBC}$ count $=4.05 \times 103 \mu \mathrm{l}$, Total WBC count $=9,800 / \mathrm{cmm}$. ESR $=04 \mathrm{~mm}$ in $1^{\text {st }}$ hour. Differential Count $=$ Neutrophil-50\%, Lymphocyte-48\%, Eosinophil-02\%, Basophil- zero, Monocyte-0\%. Urine routine examination and microscopic examination: Normal specific Gravity, Pus cells- Occasional, Renal casts and epithelial cellsoccasional. Urine Culture- No organisms isolated. 
Ultrasonography was done in full bladder showing a bulky uterus; volume: $706 \mathrm{~cm}^{3}$. The endometrial cavity was completely filled by a hypoechoic solid mass of $9.03 \times 7.64 \times 8.43 \mathrm{~cm}$; volume: $304 \mathrm{~cm}^{3}$. No intra mass degeneration or calcification was seen and diagnosed as submucosal leiomyoma (Fig. 2).

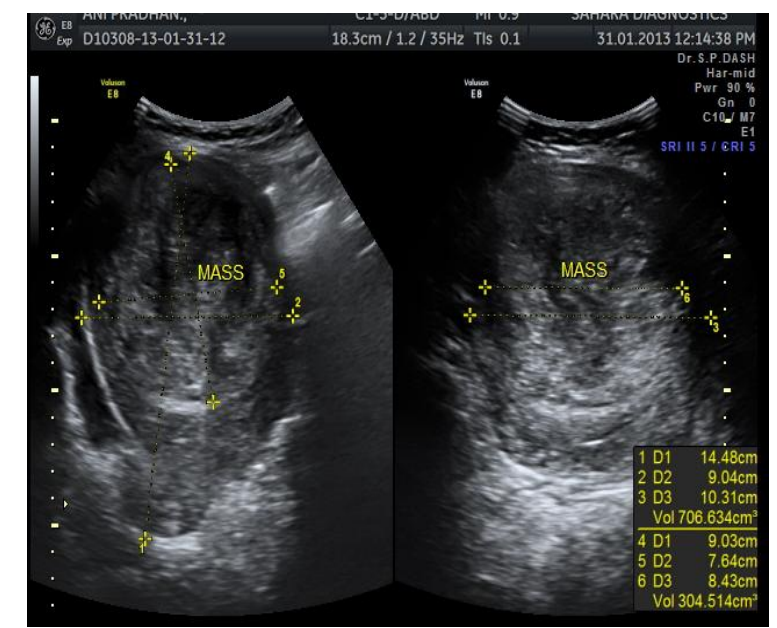

Fig. 2: USG Picture of the submucosal leiomyomata

\section{Discussion:}

Globally approximately 235 million people are affected with uterine fibroids as of 2010 (6.6\% of females $)^{[1]}$. About $20-40 \%$ of women will be diagnosed with leiomyoma at some point in their life but only a fraction of those will cause problems or require treatment. ${ }^{[2]}$. Leiomyomata are more common in obese women ${ }^{[3]}$. A lower incidence is noted in women who exercise and have lean bodies, smokers, those bearing more children and those bearing children later in life. Fibroids are dependent on estrogen and progesterone to grow and therefore relevant only during the reproductive years. They are expected to shrink after menopause.

Growth and location are the main factors that determine if a fibroid leads to symptoms and problems ${ }^{[2]}$. A small lesion can be symptomatic if located within the uterine cavity while a large lesion outside the uterus may go unnoticed. Different locations are classified as follows:

$>$ Intramural fibroids are located within the wall of the uterus and are the most common type; unless large, they may be asymptomatic. Intramural fibroids begin as small nodules in the muscular wall of the uterus. With time, intramural fibroids may expand inwards causing distortion and elongation of the uterine cavity.

$>$ Subserosal fibroids are located underneath the mucosal (peritoneal) surface of the uterus and can become very large. They can also grow out in a papillary manner to become pedunculated fibroids. These pedunculated growths can actually detach from the uterus to become a parasitic leiomyoma.

$>$ Submucosal fibroids are located in the muscle beneath the endometrium of the uterus and distort the uterine cavity; even small lesion in this location may lead to bleeding and infertility. A pedunculated lesion within the cavity is termed an intracavitary fibroid and can be passed through the cervix.

$>$ Cervical fibroids are located in the wall of the cervix (neck of the uterus). Rarely fibroids are found in the supporting structures (round ligament, broad ligament or uterosacral ligament) of the uterus that also contain smooth muscle tissue.

Fibroids may be single or multiple. Most fibroids start in an intramural location i.e., the layer of the muscle of the uterus. With further growth, some lesions may develop towards the internal cavity while others outside the uterus. Secondary changes that may develop within fibroids are hemorrhage, necrosis, calcification or cystic changes.

Each fibroid develops from a single cell in the uterine wall which undergoes transformation. The cause of this transformation is unknown but a genetic link has been established. The subsequent growth of the fibroid appears to be related to the female hormones; estrogen \& progesterone as well as other growth factors. Fibroids are common in all women but are particularly common in child bearing age of African-American women where the incidence is up to $75 \%$. They also tend to develop fibroids at an earlier age and to a greater extent.

\section{Differential Diagnosis}

Fibroids are so common that other more serious causes of abnormal bleeding or pelvic mass can often co-exist and need to be excluded. The differential diagnosis for fibroids depends on the symptoms they are causing. It can be: Dysfunctional uterine bleeding, Endometrial polyps, Endometrial carcinoma, Endometriosis, Chronic pelvic inflammatory disease, Tubo-ovarian abscess, Uterine sarcoma, Ovarian tumour, Pelvic masses 
(other causes of a pelvic mass include tumour of the large bowel, appendix abscess and diverticular abscess) or Pregnancy.

\section{1: Medical:}

\section{Treatment}

Non-steroidal anti-inflammatory agents e.g., mefenamic acid reduce menstrual blood loss and dysmenorrhoea. Antifibrinolytic agents e.g., tranexamic acid also reduce menorrhagia. The combined oral contraceptive pill is also effective if the patient requires contraception. Danazol reduces menorrhagia by suppressing gonadotrophin secretion and abolishing cyclical ovarian function. Gonadotrophin-releasing hormone (GnRH) agonists:

$>$ Produce reduction in the size of fibroids upto $50 \%$ within three months. But once discontinued, fibroids again grow to their former size within about two months. Therefore, they are mainly useful preoperatively.

$>$ They are associated with significant side-effects including amenorrhoea, menopausal symptoms and bone loss which can lead to osteoporosis in long-term use.

Most fibroids do not require treatment unless they are causing symptoms. After menopause, fibroids shrink and it is unusual for fibroids to cause problems.

\section{Symptomatic Uterine Fibroids Can Be Treated By}

$>$ medication to control symptoms

$>$ medication aimed at shrinking tumours

$>$ ultrasound fibroid destruction

$>$ myomectomy or radio frequency ablation

$>$ hysterectomy

$>$ uterine artery embolization

\section{2: Surgical:}

\section{Surgery Is Indicated When}

$>$ There is excessively enlarged uterine size.

$>$ Pressure symptoms are present.

$>$ Medical management is not sufficient to control symptoms.

$>$ Subfertility is also a factor.

\section{Surgical Options Include}

\subsection{1: Myomectomy}

> This is used in patients who wish to maintain their reproductive potential or keep their uterus. However, during surgery this may be revealed as an unrealistic and women should be consented appropriately with this in mind.

> Laparoscopic myomectomy is the best treatment option for symptomatic women with subserous fibroids, who wish to maintain their fertility ${ }^{[4]}$.

$>$ Hysteroscopic myomectomy is an established surgical procedure for women with submucosal fibroids and excessive uterine bleeding, infertility or repeated miscarriages ${ }^{[4]}$.

$>$ There is a risk of excessive bleeding and a risk of requiring hysterectomy at the time of the operation. Therefore, blood should be cross-matched preoperatively and the patient should consent (on the consent form) to hysterectomy, should the need arise.

$>$ Myomectomy carries an annual recurrence rate of $2-3 \%{ }^{[5]}$.

$>$ Pedunculated vaginal fibroids may be removed vaginally but biopsy is essential to exclude a sarcoma in women aged over 60 years.

$>$ Hysteroscopic endometrial ablation - for women presenting with menorrhagia.

$>$ MRI-guided transcutaneous focused ultrasound is safe and effective ${ }^{[6]}$. High power pulses of ultrasound are used to ablate the fibroid; there may be skin burns as a result. Long-term outcomes (fertility and need for further treatments) is uncertain as yet.

\subsection{2: Total Hysterectomy}

$>$ This has been the mainstay of treatment for many years, eliminating both symptoms and the possibility of recurrence.

$>$ In women who have completed their family, hysterectomy remains the most effective treatment for excessive uterine bleeding ${ }^{[7]}$.

$>$ It is also indicated when there are many fibroids. 
$>$ If these are small then the vaginal route is appropriate but if they are large (especially if intraligamentous) then laparotomy is indicated with preservation of ovaries if possible ${ }^{[8]}$.

$>$ The National Institute for Health and Clinical Excellence (NICE) recommends that laparoscopic techniques for hysterectomy (e.g., laparoscopically-assisted vaginal hysterectomy, laparoscopic hysterectomy, laparoscopic supracervical hysterectomy and total laparoscopic hysterectomy) appear to be sufficiently safe and effective to support their use. There is, however a higher risk of urinary tract injury and of severe bleeding in comparison with open surgery. ${ }^{[9]}$

\subsection{3: Uterine Artery Embolisation (Uae)}

$>$ This procedure has been shown to be both effective (for short and medium-term symptom relief) and safe for women who may wish to have children at some time in the future ${ }^{[10][11]}$. A register of 1,387 patients showed $84 \%$ and $83 \%$ had improvement in their symptoms at 6 and 24 months respectively.

$>$ If patients are considering pregnancy, there is a theoretical risk of placental insufficiency leading to smallfor-gestational-age babies, increased Caesarean section and prematurity.

$>$ Ensuring the tumour is a benign fibroid and not a malignant sarcoma is essential prior to $\mathrm{UAE}^{[12]}$.

$>$ Women should be informed during consenting that symptom relief may not be achieved for some women and that symptoms may return.

$>$ Compared with UAE, hysterectomy is associated with better improvement in pelvic pain. However, UAE is a good alternative to hysterectomy. ${ }^{[7]}$

The National Institute for Health and Clinical Excellence (NICE) does not currently recommend laparoscopic laser myomectomy or MRI-guided percutaneous laser ablation ${ }^{[13][14]}$.

In the present case, Total hysterectomy was done as this has been the mainstay of treatment for many years, eliminating both symptoms and the possibility of recurrence.
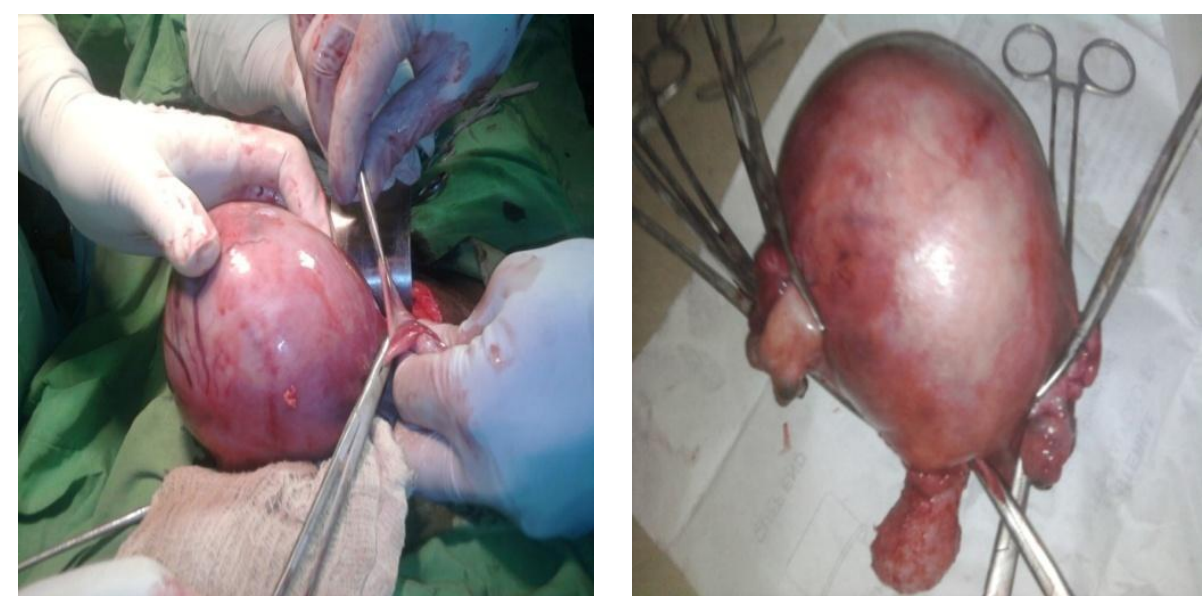

Fig. 3 shows the operative follow up Photographs
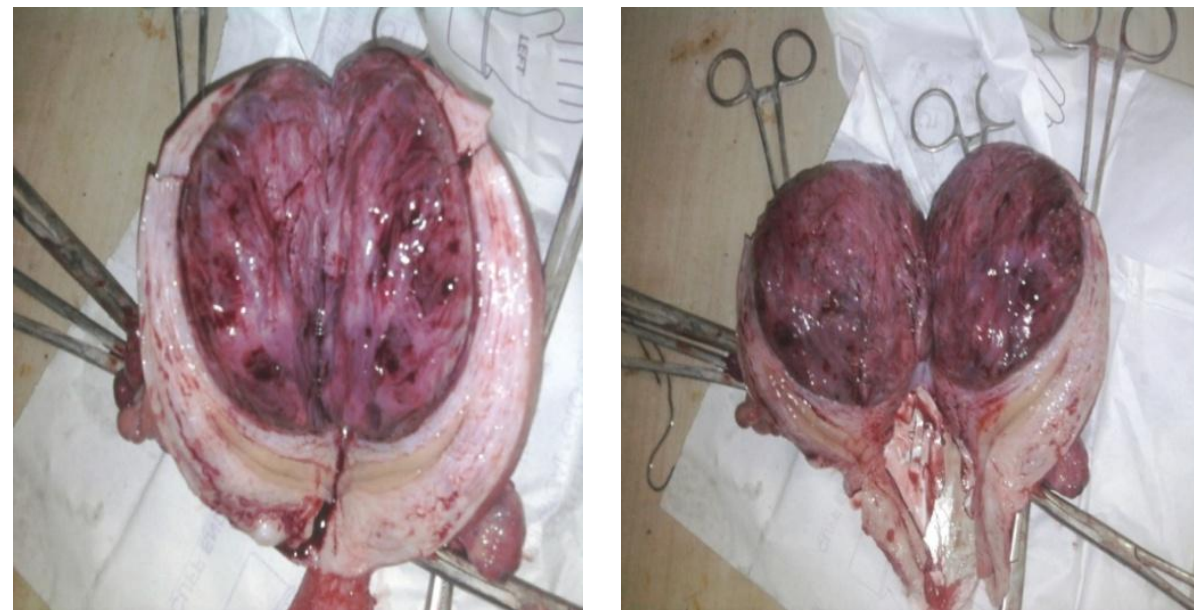

Fig. 4: following surgery and specimen cutsection

After total hysterectomy patient was kept under observation for seven days and then discharged in good condition. 


\section{Conclusion}

Fibroids are the most common benign tumors in females and typically found during the middle and later reproductive years. While most fibroids are asymptomatic, they can grow and cause heavy and painful menstruation, painful sexual intercourse, urinary frequency and urgency. Some fibroids may interfere with pregnancy although this appears to be very rare. Symptoms caused by uterine fibroids are a very frequent indication for hysterectomy. Even if minor, signs and symptoms of fibroid tumors should not be ignored.

\section{References}

[1]. Vos, T (2012 Dec 15). "Years lived with disability (YLDs) for 1160 sequelae of 289 diseases and injuries 1990-2010: a systematic analysis for the Global Burden of Disease Study 2010.". Lancet 380 (9859): 2163-96. PMID 23245607

[2]. Wallach EE, Vlahos NF (August 2004). "Uterine myomas: an overview of development, clinical features, and management". Obstet Gynecol 104 (2): 393-406. doi:10.1097/01.AOG.0000136079.62513.39. PMID 15292018

[3]. Uterine Fibroids at Merck Manual of Diagnosis and Therapy Professional Edition.

[4]. Agdi M, Tulandi T; Endoscopic management of uterine fibroids. Best Pract Res Clin Obstet Gynaecol. 2008 Mar 4;. [abstract]

[5]. Olufowobi O, Sharif K, Papaionnou S, et al; Are the anticipated benefits of myomectomy achieved in women of reproductive age? A 5-year review of the results at a UK tertiary hospital.; J Obstet Gynaecol. 2004 Jun;24(4):434-40. [abstract]

[6]. Magnetic resonance image-guided transcutaneous focused ultrasound for uterine fibroids, NICE Interventional Procedure Guideline (November 2011)

[7]. Al-Mahrizi S, Tulandi T; Treatment of uterine fibroids for abnormal uterine bleeding: myomectomy and uterine artery embolization. Best Pract Res Clin Obstet Gynaecol. 2007 Dec;21(6):995-1005. Epub 2007 May 2. [abstract]

[8]. McPherson K, Metcalfe MA, Herbert A, et al; Severe complications of hysterectomy: the VALUE study. BJOG. 2004 Jul;111(7):688-94. [abstract]

[9]. Laparoscopic techniques for hysterectomy, NICE Technology Appraisal (2007)

[10]. Uterine artery embolisation for fibroids, NICE Interventional Procedure Guideline (November 2010)

[11]. Uterine Artery Embolisation in the Management of Fibroids, Royal College of Obstetricians and Gynaecologists (Jan 2009)

[12]. Buzaglo K, Bruchim I, Lau SK, et al; Sarcoma post-embolization for presumed uterine fibroids. Gynecol Oncol. 2008 Jan;108(1):244-7. Epub 2007 Oct 22. [abstract]

[13]. Laparoscopic laser myomectomy, NICE Technology Appraisal (2003)

[14]. Magnetic resonance image-guided percutaneous laser ablation of uterine fibroids, NICE Technology Appraisal (2003) 